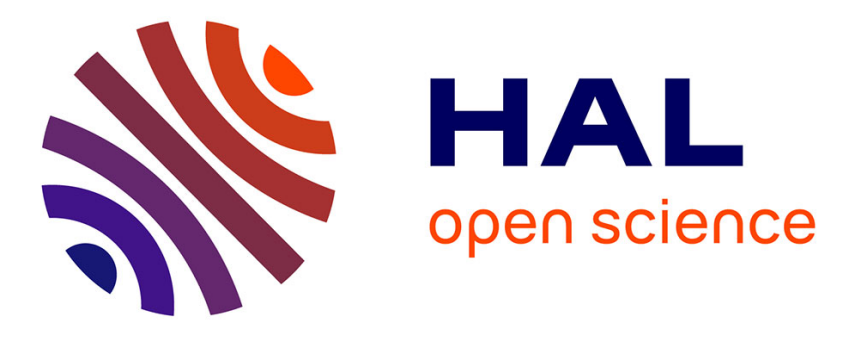

\title{
Surface-Induced Optimal Packing of Two-Dimensional Molecular Networks
}

Guillaume Copie, Fabrizio Cleri, Younes Makoudi, Christophe Krzeminski, Maxime Berthe, Frederic Cherioux, Frank Palmino, B. Grandidier

\section{- To cite this version:}

Guillaume Copie, Fabrizio Cleri, Younes Makoudi, Christophe Krzeminski, Maxime Berthe, et al.. Surface-Induced Optimal Packing of Two-Dimensional Molecular Networks. Physical Review Letters, 2015, 114 (6), pp.066101. 10.1103/PhysRevLett.114.066101 . hal-01118764

\author{
HAL Id: hal-01118764 \\ https://hal.science/hal-01118764
}

Submitted on 20 Feb 2015

HAL is a multi-disciplinary open access archive for the deposit and dissemination of scientific research documents, whether they are published or not. The documents may come from teaching and research institutions in France or abroad, or from public or private research centers.
L'archive ouverte pluridisciplinaire HAL, est destinée au dépôt et à la diffusion de documents scientifiques de niveau recherche, publiés ou non, émanant des établissements d'enseignement et de recherche français ou étrangers, des laboratoires publics ou privés. 


\title{
Surface-induced optimal packing of two-dimensional molecular networks
}

\author{
Guillaume Copie, ${ }^{1}$ Fabrizio Cleri, ${ }^{1,}{ }^{*}$ Younes Makoudi, ${ }^{2}$ Christophe Krzeminski, ${ }^{3}$ \\ Maxime Berthe, ${ }^{3}$ Frédéric Cherioux, ${ }^{2}$ Frank Palmino, ${ }^{2}$ and Bruno Grandidier ${ }^{3}$ \\ ${ }^{1}$ Université de Lille I, Institut d'Electronique Microélectronique et Nanotechnologie (IEMN), \\ CNRS, UMR 8520, Avenue Poincaré BP60069, 59652 Villeneuve d'Ascq, France \\ ${ }^{2}$ Institut FEMTO-ST, Université de Franche-Comté, CNRS, ENSMM, \\ 15B Avenue des Montboucons, F-25030 Besançon cedex, France \\ ${ }^{3}$ Institut d'Electronique Microélectronique et Nanotechnologie (IEMN), \\ CNRS, UMR 8520, Département ISEN, 41 bd Vauban, 59046 Lille, France
}

(Dated: February 20, 2015)

\begin{abstract}
High-density packing in organic crystals is usually associated with an increase of the coordination between molecules. Such a concept is not necessarily extended to two-dimensional molecular networks self-assembled on a solid surface, for which we demonstrate the key role of the surface in inducing the optimal packing. By a combination of scanning tunneling microscopy experiments and multi-scale computer simulations, we study the phase transition between two polymorphs. We find that, contrary to intuition, the structure with the lowest packing fraction corresponds to the highest molecular coordination number, due to the competition between surface and intermolecular forces. Having the lowest free energy, this structure spreads out as the most stable polymorph over a wide range of molecular concentrations.
\end{abstract}

PACS numbers: 68.43.Hn 07.79.Cz 02.70.Ns 
Organic crystals in two or three dimensions (2D, 3D) can display polymorphism because of the competition among relatively weak forces between molecules.[1] Achieving controlled and reproducible crystal structures is of particular interest since polymorph crystals might have different physical properties. Variations in color, solubility, wettability, hardness have a strong impact on potential applications. On the other hand, avoiding undesired crystallization is a major concern in manufacturing thin films, drugs or organic dyes.[1-4] Among the thermodynamic forces affecting the formation of the crystal phase (temperature, solvent, additives and impurities, template), the activity (or concentration) of free molecules is one of the primary factors. For example, concentration was found essential to account for the preferential growth of $\alpha$-glycine 3D crystals in comparison with other known polymorphs; [5] concentration was also the key parameter in the self-assembly of $2 \mathrm{D}$ molecular crystals with different geometries. [6, 7] Coupled to the direct observation of molecular patterns by scanning tunnelling microscopy (STM), manipulating the concentration has proved to be an efficient route to selectively synthesize the polymorph of choice. $[8,9]$

In this letter, we focus on the role of the surface in driving structural transitions of $2 \mathrm{D}$ molecular networks, self-assembled on a Si surface, as a function of molecule concentration. We used a combination of computer simulations and properly designed benchmark experiments to highlight the key role of the surface. Notably, all the above studies are consistent with the common wisdom that molecular packing should increase with molecule concentration.[10] As a result, upon increasing concentration one should observe structures with the highest possible packing density, since higher coordination number implies higher contact density amongst the molecules, as verified for 3D organic crystals.[11-14] However, such a straightforward consideration might be insufficient for $2 \mathrm{D}$ organic lattices assembled on a surface, because of the broken symmetry and the consequent role played by the molecule-surface interactions.

At increasing supersaturation, molecules start to cluster into structureless droplets on the surface. The loss of entropy, however, is soon outweighed by intermolecular and surface adhesion enthalpy, leading to the nucleation of ordered phases.[15]

During this self-assembly process, the interaction with the substrate can contrast the intermolecular attraction, leading to the competition and coexistence of different ordered phases at a given value of activity (the gas pressure, translating into a given number $n$ of molecules adsorbed in a finite surface patch). Since steric occupation competes with 
maximization of the coordination, the coordination of a low-density (LD) molecular network may be higher than for any other higher-density (HD) lattices, and such a LD network may become the most stable phase. In the following we give a startling example of lattices formed by planar molecules deposited on a boron-doped silicon surface for which, contrary to simple intuition, the predominant phase at high surface coverage is indeed a low-density and high-coordination one.

1,3,5-tri(4"'-cyano-4,4'-biphenyl) benzene molecules (TCNBB), consisting of three identical arms each made of two phenyl rings, attached to a central phenyl at the 1,3 and 5 carbons, and terminated by a CN group, were synthesized by a Suzuki cross-coupling reaction between adequate precursors.[16] The TCNBB molecules were subsequently evaporated at $270^{\circ} \mathrm{C}$ in ultra high vacuum onto a clean and defect-free, boron-doped $\mathrm{Si}(111)$ $(\sqrt{3} \times \sqrt{3}) R 30^{\circ}$ surface, indicated as $\mathrm{Si}: \mathrm{B}(111)$ in the following. During the evaporation, the Si crystal was maintained at room temperature. Then the surface was imaged by STM at 77 K. The deposition of TCNBB led to two different networks (Figure 1). In the center of the STM image, a low density (porous) phase is observed, whereas on the outer margins of the image, molecules appear to form a denser, compact phase with a prominent feature of parallel rows. Individual molecules are usually resolved by probing the filled electronic states. At such bias voltage, the ternary symmetry of the molecules clearly appears (Fig. 1(b), (c)). The LD phase displays a honeycomb structure, each molecule connecting three adjacent pores, defined by the end-group of six molecules. In the high-density (HD) phase, molecules are arranged in alternating parallel rows. Note that all three arms of each molecule interact with one arm of a neighbour molecule in the LD, whereas this interaction only involves one arm per molecule in the HD phase.

When the surface is fully covered with TCNBB, large-scale STM images at random locations invariably show the LD network, Fig.1(d), with only small patches of the HD coexisting in some images. By further increasing the surface coverage rate, we did not observe any additional phase transitions in the first monolayer. These experimental results indicate that the LD network is the most stable phase up to the highest coverage, at odds with recent studies of $2 \mathrm{D}$ self-assembled crystals. $[8,9]$

In order to rationalize these observations, we performed multi-scale computer simulations by means of the theoretical framework we recently developed.[15, 17] Firstly, densityfunctional theory and metadynamics (MtD) provide a best guess for the lowest-energy ad- 
sorption sites of single molecules and dimers on the surface. Then, molecular dynamics (MD) simulations using empirical force fields are performed with fully 2D-periodic molecular networks. The resulting stable structures are compared to the experimentally observed networks, finding an excellent agreement. In the case where several phases coexist, further MD simulations also allow to identify the kinetic pathway between the phases. In both the MtD and MD simulations, the MM3 force field is adopted,[18] including hydrogen bonding, and the improved description of Van der Waals forces already assessed in our previous study.[17] In practice, the infinitely-ranged VdW forces are rapidly decaying and are summed up to a cut off radius of $7 \AA$, large enough to avoid the need for a correction.[19] Finally, kinetic Monte Carlo (KMC) simulations on a frozen Si:B(111) surface, with energy parameters derived from MD, give insight into the long-time growth kinetics leading to the preferential self-assembly of the LD network.

We performed several MtD simulations [20] by placing a molecule at random sites on the surface and relaxing the system energy by steepest-descent method. The minimum-energy adsorption site coincides with the Si adatoms, as shown in Figure 2(a). Competing minima at nearly the same energy exist on the hollow sites (centers of three adatoms), separated from the adatom site by a potential barrier of about $5 \mathrm{kcal} / \mathrm{mol}$. Next, we studied the free energy landscape when a second molecule is added on the surface. The first molecule (on the left in Fig. 2(b)) is initially placed on a Si adatom; the second molecule is randomly placed within the interaction radius of the first one. By averaging over several MtD runs, the dimer configuration shown in Fig. 2(b) is obtained, with minor variations from one run to the next. In this configuration, the extra intermolecular interactions force both molecules to shift, so that their centers occupy two adjacent hollow sites.

Molecular flexibility plays an important role in stabilizing the observed networks, by allowing to optimize the molecular interactions. In Fig. 2(a) the single molecule is flat on the surface, while addition of a second molecule (Fig. 2(b)) involves a twist of the phenyl rings. By looking at the free energy differences, the hollow site in Fig. 2(a) is at $-5.5 \mathrm{kcal} / \mathrm{mol}$, while it increases to $-8.1 \mathrm{kcal} / \mathrm{mol}$ once the dimer is formed. Clearly, this difference comes entirely from the molecule-molecule interaction, including about $35 \%$ from H-bond formation and $65 \%$ from the VdW interaction, optimized by the twisting of adjacent arms.

Then, with the guidance of STM images, we built a LD and a HD network, using the 
dimer from MtD as the basic unit. The coverage rate is $n_{L D}=0.33$ and $n_{H D}=0.37$ molecules $/ \mathrm{nm}^{2}$ for the infinite (fully periodic in 2D) lattices. MD simulations were carried out on both the LD and HD networks. For both cases, the comparison of the calculated network structure against the experimental STM images is extremely good, as shown in Figure 2(c) and 2(d). After quenching down to $\mathrm{T}=0 \mathrm{~K}$, the two ordered structures differ in energy by $2.68 \pm 0.23 \mathrm{kcal} / \mathrm{mol}$. However, contrary to common wisdom, it is the LD network that yields the lowest energy. In fact, while the number of H-bonds per molecule is the same in the two lattices, the intermolecular interaction is larger for the LD network, in which all the three arms of a molecule are paired with another molecule, thereby saturating the intermolecular interactions.

By breaking down the energy contributions, we find the VdW intermolecular energy and the electrostatic energy to be lower for the LD network by $-5.1 \pm 0.3 \mathrm{kcal} / \mathrm{mol}$ and -3.1 $\mathrm{kcal} / \mathrm{mol}$ respectively, yielding an overall energy difference $\Delta E_{M M}(\mathrm{LD}-\mathrm{HD})=-8.2 \pm 0.6$ $\mathrm{kcal} / \mathrm{mol}$. Notably, the largest part of the total intermolecular energy is due to VdW forces, the average energy per molecule from H-bonds being only about $0.2 \mathrm{kcal} / \mathrm{mol}$, equal for both networks. Conversely, by looking at the molecule-surface interactions, we find a lower energy for the HD lattice, equal to $\Delta E_{M S}(\mathrm{LD}-\mathrm{HD})=+5.3 \pm 3.6 \mathrm{kcal} / \mathrm{mol}$ Since at such a low temperature the total internal energy $E(N V T)=E_{M M}+E_{M S}$ practically coincides with the free energy $G(\mu V T)$ at constant volume and chemical potential, the LD phase has indeed the lowest free energy, in agreement with the experimental STM images.

In a simple interpretation, molecule-surface interactions may be taken proportional to $n$, while molecule-molecule interactions are rather proportional to the number of contacts, $n^{2}$. Then, an energy function of the type $E(n)=\alpha n+\beta n^{2}$ should exist for any sublattice of given symmetry, at least for islands of not too large size compared to the range of molecular forces. If another phase competes with the first one, with different symmetry, coordination $\beta^{\prime}$ and surface interaction $\alpha^{\prime}$, the energy difference at zero temperature is $\Delta E(n)=(\alpha-$ $\left.\alpha^{\prime}\right) n+\left(\beta-\beta^{\prime}\right) n^{2}$. At a critical activity $n_{C}=\left(\alpha^{\prime}-\alpha\right) /\left(\beta-\beta^{\prime}\right)$, a phase transition may be observed, depending on the value of the coefficients $\alpha, \alpha^{\prime}, \beta$ and $\beta^{\prime}$.[15] Since the energy differences found in the present MD simulations (i.e., the coefficients $\left(\alpha-\alpha^{\prime}\right)$ and $\left(\beta-\beta^{\prime}\right)$ ) have opposite sign, a phase transition can occur. We can therefore guess the following kinetic phase diagram, as a function of $n$ : from $n=0$ up to $n_{C}$ denser patches of the HD phase form, initially stabilized by the predominant molecule-surface interaction; such local structures 
tend to disappear at increasing $n>n_{C}$, when the patches turn into the LD phase, because the intermolecular interaction quickly gains over the surface term; this phase persists up to $n_{L D}$, when further molecules cannot be added to the first monolayer without disrupting the symmetry.

Remarkably, the strongest intermolecular interactions correspond to the LD packing. Such a sequence in 2D crystallisation is reminiscent of Ostwald's "rule of stages",[21, 22] stating that the first crystal phase formed would be the least stable polymorph, followed by phases of increasing stability. Here, phase transformation kinetics provide a basis for this empirical rule. Note that turning a HD patch into a LD one actually increases the fraction of occupied surface. This also implies a slight increase in the fraction of broken bonds along the free perimeter of the patch, which however contribute only a rapidly vanishing $n^{1 / 2}$ surface tension term.

Since in the experiments some small patches of HD phase are sometimes observed to coexist with the predominant LD phase, we checked the possible existence of a kinetic barrier between the two structures, by using an elastic-bands MD method.[20] We find that both the intermolecular (MM) energy and the molecule-surface (MS) energy display a migration barrier in going from the HD to the LD, of $E_{M}=1.5 \mathrm{kcal} / \mathrm{mol}$ for the MM and $E_{M}=4$ $\mathrm{kcal} / \mathrm{mol}$ for the MS interaction, both quite larger than room-temperature fluctuations.[20] Therefore, patches of molecules locally arranged in the HD network could be kinetically trapped, and would not spontaneously transform into the (thermodynamically favored) LD phase, even for $n>n_{C}$, until a threshold temperature is reached

Finally, we used KMC simulations [20] to characterize the finite-temperature kinetics and long-time stability of the LD phase, in the two limiting cases of (i) homogeneous nucleation from randomly adsorbed molecules, and (ii) heterogeneous nucleation from an isolated patch of HD phase.

To study the stability of the LD phase in the case (i) of homogeneous nucleation, we ran several KMC simulations at constant- $\{\mathrm{NVT}\}$, with molecules randomly placed on the surface, at different activities and effective temperatures $k_{B} T / \Delta E \approx 1$. Up to a coverage of about 0.9 monolayer (the maximum occupancy for the LD phase, since $n_{L D} / n_{H D}=0.9$ ) KMC simulations always show the formation of a complete LD network, starting from homogeneously dispersed molecules. Upon increasing $n$ the LD network cannot grow further, and nuclei of HD appear, however without growing at any temperature. These first KMC 
simulations are consistent with our previous results, which identify the LD phase as the thermodynamically stable state.

The case of heterogeneous nucleation (ii) was also simulated by KMC at constant- $\{\mu \mathrm{VT}\}$, by including a model process of continuous deposition of molecules from the gas phase. We start the KMC simulation (Figure 3) with an isolated nucleus of HD network mimicking a $n<n_{C}$ situation, in which free molecules condensing from the gas in disordered droplets on the surface initially go into the closest energy minimum, i.e. the HD (see the above discussion about Ostwald's rule). Every $N$ timesteps $\tau$ (or effective rate $R=1 / N$ ) we add one molecule on the surface at a random position around the border of the HD patch. Between two subsequent depositions, all the molecules can move either by jumps to a neighboring site, or turn on the site in steps of $\pm 60^{\circ}$. For $R \geq 2 \times 10^{-4} \tau^{-1}$, the HD patch grows, and new HD nuclei can also be observed for the fastest rate $R=2 \times 10^{-3} \tau^{-1}$. Conversely, upon slowing down $R$ the HD phase starts to disappear; at the same time, the LD phase is nucleating and starts to grow. The effective surface diffusion coefficient is found to increase linearly with $1 / R$. Clearly, as $R$ slows down, molecules diffuse away from the HD island and nucleate the LD patches. Such findings are qualitatively consistent with the experimental results at variable activity,[20] thus lending support to our kinetic explanation about the persistence of patches of the HD within the LD phase.

In conclusion, by combining experimental STM images with multi-scale computer simulations, ranging from DFT, to molecular dynamics and metadynamics, to kinetic Monte Carlo, we studied the competition between a low-density and high-density molecular network adsorbed on a passivated $\mathrm{Si}: \mathrm{B}(111)$ surface. In agreement with the experiments, the low-density phase is the most stable one, thanks to a subtle balance between intermolecular and surface force contributions. Upon increasing the activity, the gain in molecular coordination switches the balance in favor of the former. Notably, an ordered lattice coupling lower density and higher coordination is quite a rare occurrence in $3 \mathrm{D}$ extended systems. Our results suggest that, thanks to the surface interactions, this could be a much more common finding in 2D ordered systems. 
* fabrizio.cleri@univ-lille1.fr

[1] R. J. Chesterfield, J. C. McKeen, C. R. Newman, P. C. Ewbank, D. A. da Silva Filho, J.-L. Brédas, L. L. Miller, K. R. Mann, and C. D. Frisbie, J. Phys. Chem. 108, 19281 (2004).

[2] J. Bauer, S. Spanton, R. Henry, J. Quick, W. Dziki, W. Porter, and J. Morris, Pharm. Res. 18, 859 (2001).

[3] S. Weesler, L. Lafferrére, E. Garcia, and C. Hoff, Org. Proc. Res. Dev 7, 983 (2003).

[4] J. Bernstein and E. Goldstein, Mol. Cryst. Liq. Cryst. 164, 213 (1988).

[5] Y. Yani, P. S. Chow, and R. B. H. Tan, Cryst. Growth Des. 12, 4771 (2012).

[6] S. Lei, K. Tahara, F. C. D. Schryver, M. V. der Auweraer, Y. Tobe, and S. D. Feyter, Angew. Chem. Int. Ed. 12, 4771 (2012).

[7] A. Ciesielski, P. J. Szabelski, W. Rzysko, A. Cadeddu, T. R. Cook, P. J. Stang, and P. Samori, J. Am. Chem. Soc. 135, 6942 (2013).

[8] K. Tahara, S. Okuhata, J. Adisoejoso, S. Lei, T. Fujita, S. D. Feyter, and Y. Tobe, J. Am. Chem. Soc. 131, 17583 (2009).

[9] N. T. N. Ha, T. G. Gopakumar, and M. Hietshold, J. Phys. Chem. C 115, 21743 (2011).

[10] A. I. Kitaigorodskii, Organic Chemical Crystallography (Consultants Bureau, New York, 1959).

[11] C. P. Brock and J. D. Dunitz, Chem. Mater. 6, 1118 (1994).

[12] K. E. Plass, A. L. Grzesiak, and A. J. Matzger, Acc. Chem. Res. 40, 287 (2007).

[13] M. Brinkmann, G. Gadret, M. Muccini, C. Taliani, N. Masciocchi, and A. Sironi, J. Am. Chem. Soc. 122, 5147 (2000).

[14] Z. G. Li, G. H. Wang, H. Q. Jia, N. H. Hu, and J. W. Xu, Cryst. Eng. Comm. 9, 882 (2007).

[15] F. Cleri, Phys. Rev. B 80, 235406 (2009).

[16] B. Baris, J. Jeannoutot, V. Luzet, F. Palmino, A. Rochefort, and F. Chérioux, ACS Nano 6, 6905 (2012).

[17] G. Copie, Y. Makoudi, C. Krzeminski, F. Chérioux, F. Palmino, S. Lamare, B. Grandidier, and F. Cleri, J. Phys. Chem. C 118, 12817 (2014).

[18] N. L. Allinger, Y. H. Yuh, and J. H. Lii, J. Am. Chem. Soc. 111, 8551, 8566 and 8576 (1989).

[19] M. R. Shirts, D. L. Mobley, J. D. Chodera, and V. S. Pande, J. Phys. Chem. B 111, 13052 
(2007).

[20] See Supplemental Material.

[21] W. Ostwald, Z. Phys. Chem. 22, 289 (1897).

[22] T. Threlfall, Org. Proc. Res. Dev. 7, 1017 (2003). 
FIGURES 


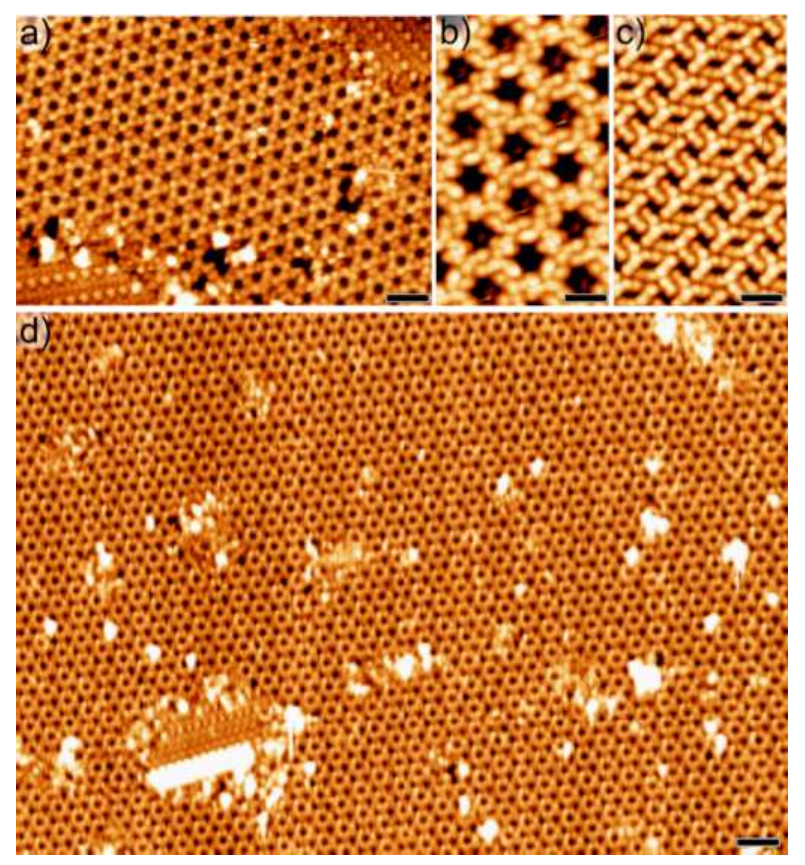

FIG. 1. (a) STM image showing both supramolecular structures obtained after the adsorption of TCNBB on a Si:B(111) surface. Tunneling conditions: $V_{S}=4.1 \mathrm{~V}, I_{t}=10 \mathrm{pA}$. (b) and (c) High resolution STM images of the porous and the compact structures, that respectively appear as a honeycomb lattice and parallel rows in (a). Tunneling conditions: $V_{S}=-2.2 \mathrm{~V} /-2.4 \mathrm{~V}, I_{t}=$ $22 \mathrm{pA} / 13 \mathrm{pA}$. (d) STM image of the Si:B(111) surface for coverage rate slightly higher than one monolayer of TCNBB. Tunneling conditions: $V_{S}=4.0 \mathrm{~V}, I_{t}=10 \mathrm{pA}$. Scale bars : (a) $5 \mathrm{~nm}$, (b) and (c) $2 \mathrm{~nm},(\mathrm{~d}) 15 \mathrm{~nm}$. 
a)
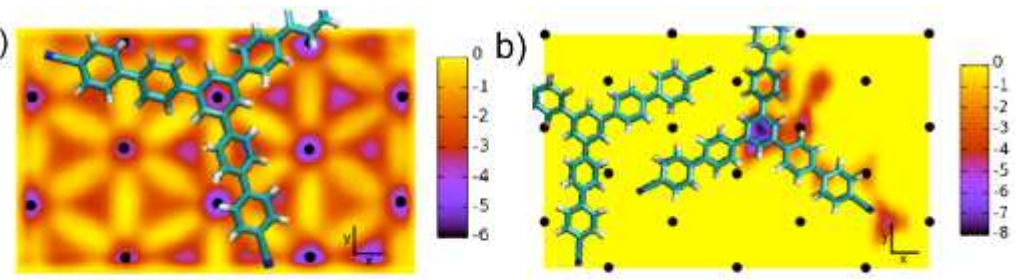

c)

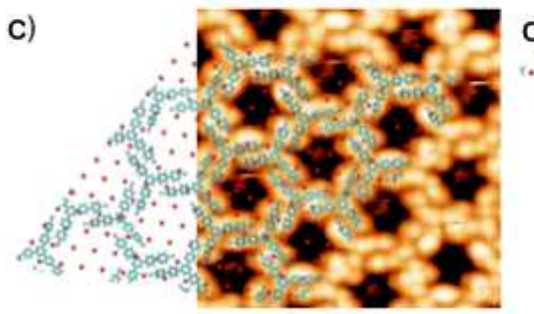

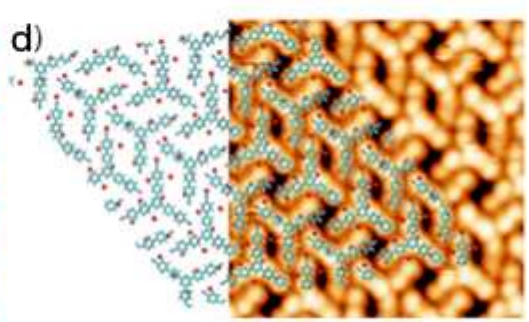

FIG. 2. Above: (a) Map of the free energy for a single TCNBB molecule adsorbed on the Si:B(111) surface. The black dots indicate the positions of the Si adatoms. (b) Excess free energy for a dimer (only the extra energy from adding the second molecule is plotted in the color map). A deep minimum at the hollow site is obtained, whence the VdW dimer interaction is maximized; energy scales in kcal/mol. Below: comparison between STM image (background) and the calculated network (foreground), after MD equilibration at $100 \mathrm{~K}$ and quenching to $\mathrm{T}=0 \mathrm{~K}$. The low-density and high density lattices are shown in (c) and (d) respectively; red dots indicate the position of the Si adatoms. 


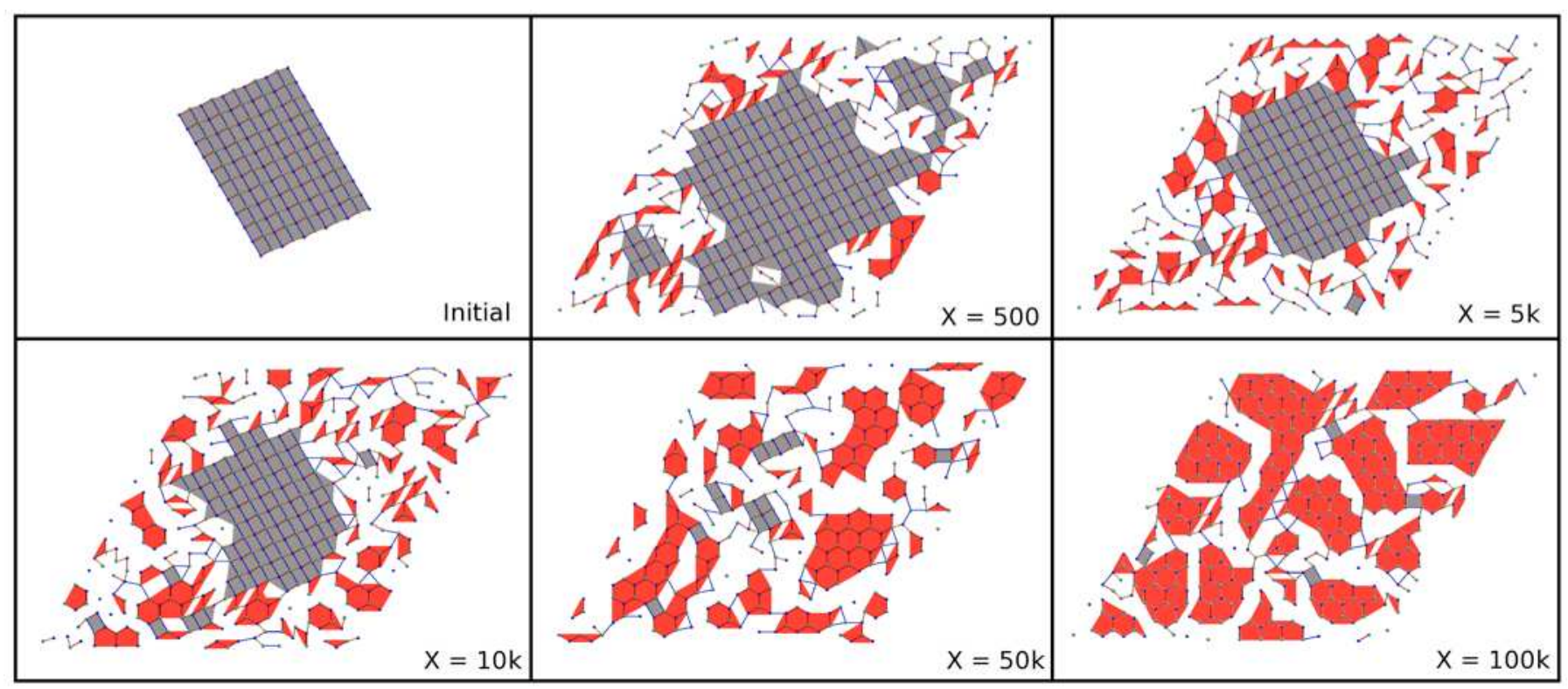

FIG. 3. Results of the KMC simulations for different rates of molecule deposition. The $\mathrm{X}$ value represents the number of steps between two successive landings. For the faster deposition rates $(\mathrm{X}=500)$ the HD network can grow (rectangular symmetry, grey shading). When slowing down the deposition rate, the HD network starts to disappear and the LD network starts to nucleate $(\mathrm{X}=5$ 10,000, hexagonal symmetry, red shading). At even slower rates $(X \geq 50,000)$, the LD network takes over the entire network. 